\title{
Smart remotely access distributed system
}

\begin{abstract}
This paper presents the data distribution called smart remotely access distributed system (SRA-DS). One of the stated purposes of the SRA-DS is to provide a standard to users in order to provide constantly available system that satisfy trust requirement for network applications. The trend in today's technology is no longer used in stand-alone units. One of the major criticisms of this feature is not constant availability. The purpose of this paper is to discuss the various aspects of monitoring and fault tolerant required for distributed system, and to describe the requirements needed in criteria from which can provide a low cost Web applications with distributed system.
\end{abstract}

Keyword: Data network; Distributed system; Remote access 